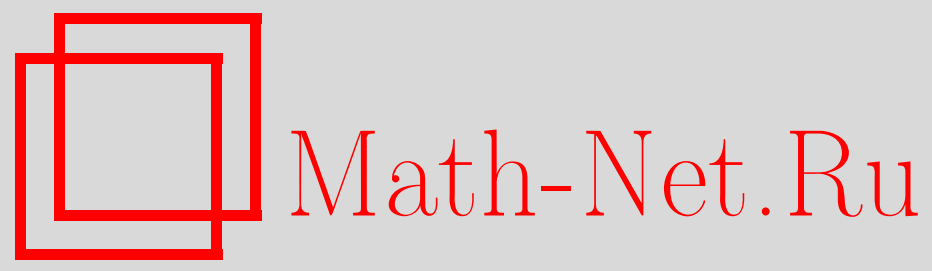

Э. К. Жимбо, В. Н. Чубариков, О распределении арифметических функций по простому модулю, Дискрет. матем., 2001, том 13, выпуск 3, 32-41

DOI: https://doi.org/10.4213/dm292

Использование Общероссийского математического портала Math-Net.Ru подразумевает, что вы прочитали и согласны с пользовательским соглашением http: //www . mathnet.ru/rus/agreement

Параметры загрузки:

IP : 3.93 .64 .190

26 апреля 2023 г., 14:49:38

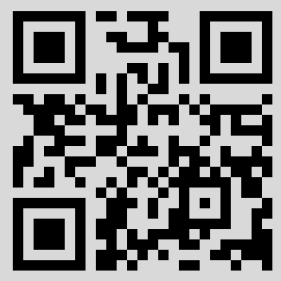




\title{
О распределении арифметических функций по простому модулю
}

\author{
(C) 2001 г. Э. К. Жимбо, В. Н. Чубариков
}

В статье рассматриваются задачи, связанные с распределением значений аналогов неполных сумм Клостермана по простому модулю и их обобщений, совместному распределению квадратичных вычетов и невычетов по разным простым модулям.

\section{1. Введение}

В настоящей работе рассматриваются задачи, которые связаны с распределением значений аналогов неполных сумм Клостермана по простому модулю и их обобщений, совместному распределению квадратичных вычетов и невычетов по разным простым модулям.

Близкой к рассматриваемым задачам является проблема И. М. Виноградова о нахождении порядка величины наименьшего квадратичного невычета по простому модулю [1-3]. Исследования И. М. Виноградова были продолжены Давенпортом и Эрдешем [4] и Берджессом [5]. В 90-е годы А. А. Карацуба [6-8] получил нетривиальные оценки аналогов неполных сумм Клостермана с весьма короткой длиной промежутка суммирования.

Другой аспект данной проблематики связан с использованием предельных теорем теории вероятностей $[4,9,11]$.

Здесь мы существенно пользуемся результатами и методами работ [1-11]. Ранее первым автором (Э. К. Жимбо) был найден закон распределения значений квадрата модуля неполных сумм Гаусса по простому модулю [12].

Обозначим через $N_{p}\{\cdot\}$ количество натуральных чисел $1 \leqslant x \leqslant p$, удовлетворяющих условиям, которые указываются в фигурных скобках.

В параграфе 2, основываясь на лемме 1 , которая по существу принадлежит А. А. Карацубе, доказываются следующие утверждения.

Теорема 1. Пусть $p-$ простое число, $h<p$ u $x$ - натуральное число. Пусть

$$
S_{p}(x ; h)=\sum_{q \leqslant h} e^{2 \pi i x q^{*} / p},
$$

где суммирование ведется по простым числам $q$ и $q^{*}$ определяетсл из сравнения

$$
q q^{*} \equiv 1 \quad(\bmod p) .
$$


Тогда при $p \rightarrow \infty, h=h(p) \rightarrow \infty, \log h / \log p \rightarrow \infty$ величина

$$
\xi=\left|\frac{S_{p}(x ; h)}{\sqrt{h}}\right|^{2}
$$

асимптотически имеет показательное распределение с параметром 1 , то есть при любом фиксированном $y>0$

$$
\lim _{p \rightarrow \infty} \frac{1}{p} N_{p}\{\xi<y\}=\int_{0}^{y} e^{-y} d y .
$$

Теорема 2. Если в условиях теоремъ 1 вместо суммъ $S_{p}(x ; h)$ рассмотретъ суммy

$$
S_{p}(a, b ; h)=\sum_{q \leqslant h} \varepsilon(q) e^{2 \pi i\left(a q+b q^{*}\right) / p},
$$

где суммирование ведется по простым числам $q,|\varepsilon(q)|=1$ для люобого простого $q$, то величина

$$
\xi=\xi(a, b)=\left|\frac{S_{p}(a, b ; h)}{\sqrt{h}}\right|^{2}
$$

асимптотически имеет похазателъное распределение с параметром 1.

В параграфе 4 с использованием свойств сумм Гаусса (лемма 1) и метода Виноградова [1] получена асимптотическая формула для количества квадратичных вычетов и невычетов в некоторых последовательностях по разным модулям. Точнее, имеет место следующее утверждение.

Теорема 3. Пусть $p_{1}, \ldots, p_{k}$ - простые числа, $p_{s} \neq p_{r}$ nрu $s \neq r, 1 \leqslant s, r \leqslant k$, $x+1 \leqslant n \leqslant x+h, \varepsilon_{s}= \pm 1$ nрu $1 \leqslant s \leqslant k$, и пусть $T-$ количество значений $n$, удовлетворяющих соотношениям

$$
\left(\frac{n+a_{1}}{p_{1}}\right)=\varepsilon_{1}, \ldots,\left(\frac{n+a_{k}}{p_{k}}\right)=\varepsilon_{k} .
$$

Tогда

$$
T=\frac{h}{2^{k}}+\theta \sqrt{Q} \ln Q
$$

где $Q=p_{1} \ldots p_{k},|\theta| \leqslant 1 u h>2^{k} \sqrt{Q}$.

Наконец, в параграфе 5 получен аналог центральной предельной теоремы теории вероятностей для распределения значений сумм символов Лежандра по разным модулям.

Теорема 4. Пусть а - положсительнал постоянная, $p_{1}, \ldots, p_{s}-$ простые числа, $s \leqslant a, Q=p_{1} \ldots p_{s}, u$ nycmb

$$
S_{h}(x)=S_{h}\left(x ; p_{1}, \ldots, p_{s}\right)=\sum_{n=x+1}^{x+h}\left(\frac{n+a_{1}}{p_{1}}\right) \ldots\left(\frac{n+a_{s}}{p_{s}}\right) .
$$

Тогда при $Q \rightarrow \infty, h=h(Q) \rightarrow \infty, \log h / \log Q \rightarrow \infty$ величина

$$
\xi_{Q}=\frac{S_{h}(x)}{\sqrt{h}}
$$

имеет асимптотически стандартное нормальное распределение.

2 Дискретная математика, т.13 №3 


\section{2. О распределении значений аналогов} неполных сумм Клостермана

Доказательство теоремъ 1. Пусть $p$ - простое число, $h<p$ и $x$ - натуральное число. Рассмотрим сумму

$$
S_{p}(x ; h)=\sum_{q \leqslant h} e^{2 \pi i x q^{*} / p}
$$

где суммирование ведется по простым числам $q$ и $q^{*}$ определяется из сравнения

$$
q q^{*} \equiv 1 \quad(\bmod p) .
$$

Для того чтобы в дальнейшем проводить теоретико-вероятностную аналогию, положим

$$
\xi=\xi_{p}(x)=\left|\frac{S_{p}(x)}{\sqrt{h}}\right|^{2}=\frac{\left|S_{p}(x)\right|^{2}}{h},
$$

и предположим, что любое $x$ с условием $1 \leqslant x \leqslant p$ принимается с одинаковой вероятностью $1 / p$. Тогда момент порядка $r$ случайной величины $\xi_{p}(x)$ будет равен

$$
M \xi_{p}^{r}=A_{p}(r)=\frac{1}{p} \sum_{x=1}^{p} \xi_{p}^{r}(x)=\frac{1}{p h^{r}} \sum_{x=1}^{p}\left|\sum_{q \leqslant h} e^{2 \pi i x q^{*} / p}\right|^{2 r} .
$$

Обозначим через $T(h)=T_{p}(h)$ число решений сравнения

$$
q_{1}^{*}+\ldots+q_{r}^{*}-q_{r+1}^{*}-\ldots-q_{2 r}^{*} \equiv 0 \quad(\bmod p) .
$$

Ясно что

$$
A_{p}(r)=\frac{1}{p h^{r}} \sum_{1 \leqslant q_{1}, \ldots, q_{2 r} \leqslant h} \sum_{x=1}^{p} e^{2 \pi i x\left(q_{1}^{*}+\ldots+q_{r}^{*}-q_{r+1}^{*}-\ldots-q_{2 r}^{*}\right) / p}=h^{-r} T(h) .
$$

Здесь мы воспользовались тем, что

$$
\frac{1}{p} \sum_{x=1}^{p} e^{2 \pi i x m / p}=\left\{\begin{array}{lll}
1, & \text { если } m \equiv 0 & (\bmod p), \\
0, & \text { если } m \neq \equiv 0 & (\bmod p) .
\end{array}\right.
$$

Найдем асимптотическую формулу для величины $T(h)$ при $h<p$ и $h \rightarrow \infty$. Справедливо следующее утверждение.

Лемма 1. Пусть $q_{1}, \ldots, q_{r}, q_{r+1}, \ldots, q_{2 r}-$ простье числа, не превосходящие $h, u$ $h^{2 r-1}<(2 r)^{-1} p$. Тогда для числа $T(h)=T_{p}(h)$ решений сравнения

$$
q_{1}^{*}+\ldots+q_{r}^{*}-q_{r+1}^{*}-\ldots-q_{2 r}^{*} \equiv 0 \quad(\bmod p)
$$

при $h \rightarrow \infty$ имеет место асимптотическая формула

$$
T(h)=r ! h^{r}+O\left(h^{r-1}\right) .
$$


Доказательство. Домножив сравнение (1) на

$$
Q=q_{1} \ldots q_{r} q_{r+1} \ldots q_{2 r} \not \equiv 0 \quad(\bmod p)
$$

получим сравнение

$$
\begin{aligned}
& q_{2} \ldots q_{r} q_{r+1} \ldots q_{2 r}+\ldots+q_{1} \ldots q_{r-1} q_{r+1} \ldots q_{2 r} \\
& \quad-q_{1} \ldots q_{r+1} q_{r+2} \ldots q_{2 r}-\ldots-q_{1} \ldots q_{r} q_{r+1} \ldots q_{2 r-1} \equiv 0 \quad(\bmod p) .
\end{aligned}
$$

Поскольку каждое слагаемое в этом сравнении меньше, чем $p /(2 r)$, оно будет уравнением

$$
\frac{Q}{q_{1}}+\ldots+\frac{Q}{q_{r}}-\frac{Q}{q_{r+1}}-\ldots-\frac{Q}{q_{2 r}}=0
$$

Наборы $\left(q_{r+1}, \ldots, q_{2 r}\right)$, которые являются перестановкой наборов $\left(q_{1}, \ldots, q_{r}\right)$, являются решениями последнего уравнения. Их количество $T_{1}$ выражается формулой

$$
T_{1}=r ! h^{r}+O\left(h^{r-1}\right) .
$$

Оценим сверху число решений $\left(q_{1}, \ldots, q_{r}, q_{r+1}, \ldots, q_{2 r}\right)$, для которых $\left(q_{r+1}, \ldots, q_{2 r}\right)$ не является перестановкой набора $\left(q_{1}, \ldots, q_{r}\right)$. Без ограничения общности можно считать, что $q_{1} \leqslant \ldots \leqslant q_{r}$ и $q_{r+1} \leqslant \ldots \leqslant q_{2 r}$.

Пусть $q-$ максимальное из чисел $q_{s}$ и $q_{r+s}$ такое, что

$$
q_{2 r}=q_{r}, \ldots, q_{r+s+1}=q_{s+1}, q_{r+s} \neq q_{s}
$$

Тогда уравнение (2) можно переписать в виде

$$
\frac{Q}{q_{1}}+\ldots+\frac{Q}{q_{s}}=\frac{Q}{q_{r+1}}+\ldots+\frac{Q}{q_{r+s}} .
$$

Рассмотрим две возможности: $q>r$ и. $q \leqslant r$.

В первом случае все слагаемые в (3), кроме $q_{m}=q$, делятся на $q$. Заметим также, что все слагаемые, отвечающие $q_{m}=q$, находятся только в одной части равенства (3). Следовательно, для того чтобы выполнялось равенство (3) необходимо, чтобы количество слагаемых, отвечающих $q_{m}=q$, делилось на $q$. Отсюда следует, что это количество не меньше $q>r$. Но так как в одной стороне равенства находится ровно $r$ слагаемых, случай не имеет места.

Рассмотрим теперь случай $q \leqslant r$. В этом случае $s \geqslant 1$ и уравнение (3) имеет не более $r^{2 s}$ решений. Отсюда следует, что уравнению (2) удовлетворяют не более $r^{2 s} h^{r-s}<h^{r-1}$ наборов. Лемма доказана.

Продолжим доказательство теоремы 1 . Для любого фиксированного $r$ при $p \rightarrow \infty$

$$
h \rightarrow \infty, \quad \frac{\log h}{\log p} \rightarrow 0,
$$

следовательно, при $p \rightarrow \infty$

$$
A_{p}(r) \rightarrow r ! .
$$

Поэтому предельное распределение $\xi_{p}$ - это показательное распределение, поскольку его момент порядка $r \geqslant 1$ равен $r$ !. Теорема 1 доказана. 


\section{3. О распределении значений обобщенных неполных сумм Клостермана}

Доказателъство теоремъ 2. Пусть, как и прежде, $p$ - простое число, $h<p$ и $a, b$ - натуральные числа. Рассмотрим сумму

$$
S_{p}(a, b ; h)=\sum_{q \leqslant h} \varepsilon(q) e^{p \pi i\left(a q+b q^{*}\right) / p},
$$

где суммирование ведется по простым числам $q,|\varepsilon(q)|=1$ для любого простого $q$. Такие суммы мы будем называть обобщенными неполными суммами Клостермана.

Пусть

$$
\xi=\xi_{p}(a, b)=\left|\frac{S_{p}(a, b ; h)}{\sqrt{h}}\right|^{2}=\frac{\left|S_{p}(a, b ; h)\right|^{2}}{h} .
$$

Предположим, что любой набор $(a, b)$ с условием $1 \leqslant a, b \leqslant p$ принимается с одинаковой вероятностью $1 / p^{2}$. Тогда момент порядка $r$ случайной величины $\xi_{p}(a, b)$ будет равен

$$
\begin{aligned}
A_{p}(r)= & \frac{1}{p^{2} h^{r}} \sum_{a=1}^{p} \sum_{b=1}^{p}\left|\sum_{q \leqslant h} \varepsilon(q) e^{2 \pi i\left(a q+b q^{*}\right) / p}\right|^{2 r} \\
= & \frac{1}{p^{2} h^{r}} \sum_{1 \leqslant q_{1}, \ldots, q_{2 r} \leqslant h} \varepsilon\left(q_{1}\right) \ldots \varepsilon\left(q_{r}\right) \bar{\varepsilon}\left(q_{r+1}\right) \ldots \bar{\varepsilon}\left(q_{2 r}\right) \\
& \quad \times \sum_{a=1}^{p} e^{2 \pi i a\left(q_{1}+\ldots+q_{r}-q_{r+1}-\ldots-q_{2 r}\right) / p} \sum_{b=1}^{p} e^{2 \pi i b\left(q_{1}^{*}+\ldots+q_{r}^{*}-q_{r+1}^{*}-\ldots-q_{2 r}^{*}\right) / p} \\
= & h^{-r} T(h ; a, b),
\end{aligned}
$$

где $T(h ; a, b)$ - количество решений системы сравнений

$$
\begin{aligned}
q_{1}+\ldots+q_{r}-q_{r+1}-\ldots-q_{2 r} \equiv 0 & (\bmod p), \\
q_{1}^{*}+\ldots+q_{r}^{*}-q_{r+1}^{*}-\ldots-q_{2 r}^{*} \equiv 0 & (\bmod p) .
\end{aligned}
$$

Отбросив первое сравнение этой системы и воспользовавшись леммой 1 , получим, что

$$
A_{p}(r)=r !+O\left(h^{-1}\right) \text {. }
$$

Отсюда, переходя к пределу при $p \rightarrow \infty$, находим, что

$$
A_{p}(r) \rightarrow r ! .
$$

Поэтому величина $\xi_{p}=\left|S_{p}(a, b ; h) / \sqrt{h}\right|^{2}$ имеет показательное распределение с параметром $\lambda$, равным 1 . Теорема 2 доказана.

\section{4. Оценка арифметических сумм в классах вычетов по различным модулям}

Лемма 2. Пусть а-целое число,

$$
U_{a, p}=\sum_{x=1}^{p-1}\left(\frac{x}{p}\right) e^{2 \pi i a x / p}
$$


Tогда

$$
\left(\frac{a}{p}\right)=\frac{U_{a, p}}{U_{1, p}}
$$

a $\operatorname{npu}(a, p)=1$

$$
\left|U_{a, p}\right|=\sqrt{p} .
$$

Доказательство см. [3], стр. 80-81, вопрос 11a.

Лемма 3. Пусть $p_{1}, \ldots, p_{s}$ - различные простье числа, $Q=p_{1} \ldots p_{s} u a_{1}, \ldots, a_{s}$ - произвольные челые числа. Тогда для суммы

$$
S=\sum_{n=x+1}^{x+h}\left(\frac{n+a_{1}}{p_{1}}\right) \ldots\left(\frac{n+a_{s}}{p_{s}}\right)
$$

справедлива оценка

$$
|S| \leqslant \sqrt{Q} \log Q
$$

Доказательство. По лемме 2 для суммы Гаусса при некотором $\varepsilon_{p_{j}},\left|\varepsilon_{p_{j}}\right|=1$, справедливо равенство

$$
\left(\frac{n+a_{j}}{p_{j}}\right)=\frac{\varepsilon_{p_{j}}}{\sqrt{p_{j}}} \sum_{m \leqslant p_{j}}\left(\frac{m}{p_{j}}\right) e^{-2 \pi i m\left(n+a_{j}\right) / p_{j}}, \quad j=1, \ldots, s
$$

поэтому

$$
\begin{aligned}
S= & \sum_{n=x+1}^{x+h} \frac{\varepsilon_{p_{1}}}{\sqrt{p_{1}}} \sum_{m_{1}=1}^{p_{1}-1}\left(\frac{m_{1}}{p_{1}}\right) e^{-2 \pi i m_{1}(n+a) / p_{1}} \ldots \frac{\varepsilon_{p_{s}}}{\sqrt{p_{s}}} \sum_{m_{s}=1}^{p_{s}-1}\left(\frac{m_{s}}{p_{s}}\right) e^{-2 \pi i m_{s}\left(n+a_{s}\right) / p_{s}} \\
= & \frac{\varepsilon_{p_{1}} \ldots \varepsilon_{p_{s}}}{\sqrt{p_{1} \ldots p_{s}}} \sum_{m_{1}=1}^{p_{1}-1}\left(\frac{m_{1}}{p_{1}}\right) e^{-2 \pi i m_{1}\left(n+a_{1}\right) / p_{1}} \ldots \sum_{m_{s}=1}^{p_{s}-1}\left(\frac{m_{s}}{p_{s}}\right) e^{-2 \pi i m_{s}\left(n+a_{s}\right) / p_{s}} \\
& \quad \times \sum_{n=x+1}^{x+h} e^{-2 \pi i n\left(m_{1} / p_{1}+\ldots+m_{s} / p_{s}\right)}
\end{aligned}
$$

Отсюда следует оценка

$$
\begin{aligned}
|S| & \leqslant \frac{1}{\sqrt{Q}} \sum_{m_{1}=1}^{p_{1}-1} \ldots \sum_{m_{s}=1}^{p_{s}-1}\left|\sum_{n=x+1}^{x+h} e^{2 \pi i n\left(m_{1} / p_{1}+\ldots+m_{s} / p_{s}\right)}\right| \\
& =\frac{1}{\sqrt{Q}} \sum_{1 \leqslant l \leqslant Q}^{\prime}\left|\sum_{n=x+1}^{x+h} e^{2 \pi i n l / Q}\right|=T,
\end{aligned}
$$

где штрих в знаке суммы означает суммирование по $l, 1 \leqslant l \leqslant Q$, взаимно простым с $Q$.

Здесь мы воспользовались тем, что если $m_{r}, 1 \leqslant r \leqslant s$, пробегает приведенную систему вычетов по модулю $p_{r}$ и

$$
\frac{m_{1}}{p_{1}}+\ldots+\frac{m_{s}}{p_{s}}=\frac{l}{Q}, \quad Q=p_{1} \ldots p_{s}
$$


то $l$ пробегает полную систему вычетов по модулю $Q$.

Далее, находим, что

$$
\begin{aligned}
|T| & \leqslant \frac{2}{\sqrt{Q}} \sum_{0 \leqslant l \leqslant \frac{Q}{2}}^{\prime}\left|\frac{e^{2 \pi i l / Q}-e^{2 \pi i l(h+1) / Q}}{1-e^{2 \pi i l / Q}}\right| \\
& =\frac{2}{\sqrt{Q}} \sum_{0 \leqslant l \leqslant Q / 2}^{\prime}\left|\frac{\sin (\pi l h / Q)}{\sin (\pi l / Q)}\right| \leqslant \frac{1}{\sqrt{Q}} \sum_{0 \leqslant l \leqslant Q / 2}^{\prime} \frac{Q}{l}<\sqrt{Q} \log Q .
\end{aligned}
$$

Лемма 3 доказана.

Доказательстьо теоремъ 3. Очевидно, что

$$
\begin{aligned}
\frac{1}{2^{k}}\left(1-\varepsilon_{1}\left(\frac{n+a_{1}}{p_{1}}\right)\right) \ldots\left(1-\varepsilon_{k}\left(\frac{n+a_{k}}{p_{k}}\right)\right) \\
\quad= \begin{cases}1, & \text { если }\left(\frac{n+a_{1}}{p_{1}}\right)=\varepsilon_{1}, \ldots,\left(\frac{n+a_{k}}{p_{k}}\right)=\varepsilon_{k}, \\
0 & \text { в противном случае. }\end{cases}
\end{aligned}
$$

Рассмотрим выражение

$$
\begin{aligned}
a(n)=\frac{1}{2^{k}} \prod_{s=1}^{k}\left(1-\varepsilon_{s}\left(\frac{n+a_{s}}{p_{s}}\right)\right) & \\
& =\frac{1}{2^{k}}+\frac{1}{2^{k}} \sum_{s_{1}, \ldots, s_{l}}(-1)^{l} \varepsilon_{s_{1}} \ldots \varepsilon_{s_{l}}\left(\frac{n+a_{s_{1}}}{p_{s_{1}}}\right) \ldots\left(\frac{n+a_{s_{l}}}{p_{s_{l}}}\right) .
\end{aligned}
$$

Просуммировав величины $a(n)$ по $n$ от 1 до $h$, получим, что

$$
\sum_{n=x+1}^{x+h} a(n)=\frac{h}{2^{k}}+\frac{\theta}{2^{k}} \sum_{s_{1}, \ldots, s_{l}} \sqrt{p_{s_{1}} \ldots p_{s_{l}}} \log \left(p_{s_{1}} \ldots p_{s_{l}}\right)=\frac{h}{2^{k}}+\frac{\theta \ln Q}{2^{k}} \prod_{s=1}^{k}\left(1+\sqrt{p_{s}}\right) .
$$

Поскольку $1+\sqrt{p_{s}} \leqslant 2 \sqrt{p_{s}}$, последнее выражение равно

$$
\frac{h}{2^{k}}+\theta_{0} \sqrt{Q} \ln Q
$$

где $\left|\theta_{0}\right| \leqslant 1$ - некоторая постоянная.

Теорема 3 доказана.

\section{5. О совместном распределении арифметических последовательностей по нескольким модулям}

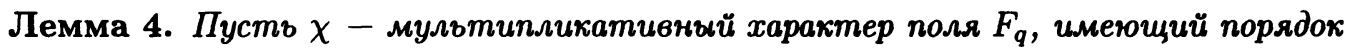
$m>1$, и пусть $f \in F_{q}[x]$ - нормированный многочлен положстельной степени, не являющийся $m$-й степенъю другого многочлена. Если $d$ - количество различных корней многочлена $f$ в его поле разложсения над $F_{q}$, то для кажсдого $a \in F_{q}$ выполняется неравенство

$$
\left|\sum_{c \in F_{q}} \chi(a f(c))\right| \leqslant(d-1) \sqrt{q}
$$


Доказательство можно найти, например, в [10], стр. 279.

Перейдем к доказательству теоремы 4. Пусть $p_{1}, \ldots, p_{s}$ - простые числа. Рассмотрим сумму

$$
S_{h}(x)=S_{h}\left(x ; p_{1}, \ldots, p_{s}\right)=\sum_{n=x+1}^{x+h}\left(\frac{n+a_{1}}{p_{1}}\right) \ldots\left(\frac{n+a_{s}}{p_{s}}\right) .
$$

Вычислим сначала момент порядка $2 r$ величины

$$
\xi=\xi_{p_{1}, \ldots, p_{s}}=\frac{S_{h}(x)}{\sqrt{h}} .
$$

Справедливы равенства

$$
\begin{aligned}
A_{p_{1}, \ldots, p_{s}}(2 r) & =\frac{1}{p_{1} \ldots p_{s} h^{r}} \sum_{x=1}^{p_{1} \ldots p_{s}}\left(\sum_{n=x+1}^{x+h}\left(\frac{n+a_{1}}{p_{1}}\right) \ldots\left(\frac{n+a_{s}}{p_{s}}\right)\right)^{2 r} \\
& =\frac{1}{p_{1} \ldots p_{s} h^{r}} \sum_{x=1}^{p_{1} \ldots p_{s}}\left(\sum_{n=1}^{h}\left(\frac{n+x+a_{1}}{p_{1}}\right) \ldots\left(\frac{n+x+a_{s}}{p_{s}}\right)\right)^{2 r} \\
& =\frac{1}{p_{1} \ldots p_{s} h^{r}} \sum_{n_{1}, \ldots, n_{2}=1}^{h}\left(\frac{f_{1}(x ; \bar{n})}{p_{1}}\right) \ldots\left(\frac{f_{s}(x ; \bar{n})}{p_{s}}\right)
\end{aligned}
$$

где $\bar{n}=\left(n_{1}, \ldots, n_{2 r}\right)$ и

$f_{1}(x ; \bar{n})=\left(x+n_{1}+a_{1}\right) \ldots\left(x+n_{2 r}+a_{1}\right), \ldots, f_{s}(x ; \bar{n})=\left(x+n_{1}+a_{s}\right) \ldots\left(x+n_{2 r}+a_{s}\right)$.

По китайской теореме об остатках, если

$$
x \equiv Q p_{1}^{-1} x_{1}+\ldots+Q p_{s}^{-1} x_{s} \quad(\bmod Q), \quad Q=p_{1} \ldots p_{s}
$$

и $x$ пробегает полную систему вычетов по модулю $Q$, то $x_{t}$ пробегает полную систему вычетов по модулю $p_{t}, t=1, \ldots, s$. Поэтому последняя сумма равна

$$
\frac{1}{Q h^{r}} \sum_{n_{1}, \ldots, n_{2 r}=1}^{h} \sum_{x_{1}=1}^{p_{1}}\left(\frac{f_{1}\left(Q p_{1}^{-1} x_{1} ; \bar{n}\right)}{p_{1}}\right) \ldots \sum_{x_{r}=1}^{p_{r}}\left(\frac{f_{s}\left(Q p_{s}^{-1} x_{s} ; \bar{n}\right)}{p_{s}}\right),
$$

где

$$
f_{t}\left(Q p_{t}^{-1} x_{t} ; \bar{n}\right)=\left(n_{1}+Q p_{t}^{-1} x_{t}+a_{t}\right) \ldots\left(n_{2 r}+Q p_{t}^{-1} x_{t}+a_{t}\right), \quad t=1, \ldots, s .
$$

Отсюда получаем, что

$$
A_{p_{1}, \ldots, p_{s}}(2 r)=\frac{1}{p_{1} \ldots p_{s} h^{r}} \sum_{n_{1}, \ldots, n_{2 r}=1}^{h} \prod_{t=1}^{s} \sum_{x_{t}=1}^{p_{t}}\left(\frac{f_{t}\left(Q p_{t}^{-1} x_{t} ; \bar{n}\right)}{p_{t}}\right) .
$$

Далее, разобъем наборы натуральных чисел $\left(n_{1}, \ldots, n_{2 r}\right)$ на два класса.

Прежде всего заметим следующее. Так как $Q=p_{1} \ldots p_{s}$, при некотором $t$, $1 \leqslant t \leqslant s$, существует $p=p_{t}$ такое, что $p \geqslant Q^{1 / s} \geqslant Q^{1 / a}$. Поскольку $\log h / \log Q \rightarrow 0$ 
при $Q \rightarrow \infty$, можно считать, что при достаточно большом $Q$ выполняется неравенство $Q^{1 / a}>h$.

В первый класс отнесем те и только те наборы $\left(n_{1}, \ldots, n_{2 r}\right)$, которые состоят из не более чем $r$ различных натуральных чисел, каждое из которых встречается четное число раз. Оставшиеся наборы отнесем ко второму классу. В соответствии с этим разбиением сумму $A_{p_{1}, \ldots, p_{s}}(2 r)$ представим в виде

$$
A_{p_{1}, \ldots, p_{s}}(2 r)=B_{1}+B_{2}
$$

где в сумму $B_{1}$ входят наборы первого класса, а в сумму $B_{2}$ - наборы второго класса.

Для наборов первого класса по любому модулю $p_{t}, 1 \leqslant t \leqslant s$, многочлены $f_{t}\left(Q p_{t}^{-1} x_{t}\right)$ представляют собой точный квадрат по модулю $p_{t}$. Следовательно, при любом $t, 1 \leqslant t \leqslant s$, сумма

$$
\sum_{x_{t}=1}^{p_{t}}\left(\frac{f_{t}\left(Q p_{t}^{-1} x_{t} ; \bar{n}\right)}{p_{t}}\right)
$$

равна $p_{t}-\theta_{1} t$ при некотором $\theta_{1}, 0 \leqslant \theta_{1} \leqslant 1$.

Для наборов второго класса по лемме 4 при выбранном выше $p$ справедлива оценка

$$
\left|\sum_{x_{t}=1}^{p_{t}}\left(\frac{f_{t}\left(Q p_{t}^{-1} x_{t} ; \bar{n}\right)}{p_{t}}\right)\right| \leqslant r \sqrt{p}
$$

Следовательно,

$$
\begin{aligned}
& B_{1}=\frac{1}{Q h^{r}}\left((2 r-1) ! ! h^{r}(Q-\theta r)+O\left(p h^{r-1}\right)\right), \\
& B_{2} \leqslant \frac{1}{Q h^{r}} h^{2 r} Q p^{-1} r \sqrt{p} \leqslant h^{r} Q^{-1 /(2 a)} .
\end{aligned}
$$

При оценке $A_{p_{1}, \ldots, p_{s}}(2 r-1)$ первый класс будет пустым и $B_{1}=0$, а для второго класса имеет место та же оценка величины $B_{2}$.

Таким образом, в зависимости от четности порядка момента величины $\xi$ получаем оценки

$$
\begin{aligned}
A_{p_{1}, \ldots, p_{s}}(2 r) & =1 \cdot 3 \ldots(2 r-1)+O\left(h^{-1}\right), \\
A_{p_{1}, \ldots, p_{s}}(2 r-1) & \left.\ll h^{r} Q^{-1 /(2 a)}\right) .
\end{aligned}
$$

Таким образом, при $Q \rightarrow \infty$

$$
\begin{aligned}
A_{p}(2 r) & \rightarrow(2 r-1) ! ! \\
A_{p}(2 r-1) & \rightarrow 0
\end{aligned}
$$

здесь и выше $(2 r-1) ! !=1 \cdot 3 \ldots(2 r-1)$. Следовательно, величина $\xi_{Q}=S_{h}(x) / \sqrt{h}$ при $Q \rightarrow \infty$ имеет в пределе стандартное нормальное распределение. Теорема 4 доказана. 


\section{Список литературы}

1. Виноградов И. М., Sur la distribution des residues et des nonresidues des puissances. Жypнал физ.-матем. об-ва при Пермском ун-те (1918) 1, 94-98.

2. Виноградов И. М., О распределении квадратичных вычетов и невычетов. Журнал физ.матем. об-ва при Пермском ун-те (1919) 2, 1-16.

3. Виноградов И. М., Основы теории чисел. Наука, Москва, 1972.

4. Davenport H., Erdös P., The distribution of quadratic and higher residues. Publ. Math. Debrecen (1952) 2, Ne3-4, 252-265.

5. Burgess D. A., The distribution of quadratic residues and nonresidues. Math. (1957) 4, №8, 106-112.

6. Карацуба А. А., Распределение обратных величин в кольце вычетов по заданному модулю. Докл. РАН. (1993) 333, №2, 138-139.

7. Карацуба А. А., Аналоги сумм Клостермана. Изв. РАН. Сер. матем. (1995) 59, №5, 93-102.

8. Карацуба А. А., Двойные суммы Клостермана. Матем. заметки (1999) 66, №5, 682687.

9. Кубилюс И. П., Линник Ю. В., Арифметическое моделирование броуновского движения. Изв. вузов. Математика (1959) 13, 88-95.

10. Лидл Р., Нидеррайтер Г., Конечные поля, т. 1. Мир, Москва, 1988.

11. Линник Ю. В., Эргодические свойства алгебраических полей. Наука, Ленинград, 1967.

12. Жимбо Э. К., О распределении значений модулей неполных сумм Гаусса. В сб.: Тезисы Межсдународной конф. "Современное состояние и перспективы развития математики в рамках программы «Казахстан в третъем тысячелетии»". Алматы, 26-28 октября 2000, с. 80.

Статья поступила 18.12.2000. 